\title{
Assessing the Population-Level Correlation of Medication Regimen Complexity and Adherence Indices Using Electronic Health Records and Insurance Claims
}

\author{
Xiaomeng Ma, MS; Changmi Jung, PhD; Hsien-Yen Chang, PhD; \\ Thomas M. Richards, MS; and Hadi Kharrazi, MD, PhD
}

\begin{abstract}
BACKGROUND: Nonadherence to medication regimens can lead to adverse health care outcomes and increasing costs.

OBJECTIVES: To (a) assess the level of medication complexity at an outpatient setting using population-level electronic health record (EHR) data and (b) evaluate its association with medication adherence measures derived from medication-dispensing claims.
\end{abstract}

METHODS: We linked EHR data with insurance claims of 70,054 patients who had an encounter with a U.S. midwestern health system between 2012 and 2013. We constructed 3 medication-derived indices: medication regimen complexity index (MRCI) using EHR data; medication possession ratio (MPR) using insurance pharmacy claims; and prescription fill rates (PFR; 7 and 30 days) using both data sources. We estimated the partial correlation between indices using Spearman's coefficient (SC) after adjusting for age and sex.

RESULTS: The mean age (SD) of 70,054 patients was 37.9 (18.0) years, with an average Charlson Comorbidity Index of 0.308 (0.778). The 2012 data showed mean (SD) MRCI, MPR, and 30-day PFR of 14.6 (17.8), 0.624 $(0.310)$, and $81.0(27.0)$, respectively. Patients with previous inpatient stays were likely to have high MRCI scores (36.3 [37.9], $P<0.001)$ and were less adherent to outpatient prescriptions (MPR $=50.3$ [27.6\%], $P<0.001$; 30-day PFR $=75.7$ [23.6\%], $P<0.001)$. However, MRCI did not show a negative correlation with MPR $(S C=-0.31, P<0.001)$ or with 30 -day PFR $(\mathrm{SC}=-0.17, P<0.001)$ at significant levels.

CONCLUSIONS: Medication complexity and adherence indices can be calculated on a population level using linked EHR and claims data. Regimen complexity affects patient adherence to outpatient medication, and strength of correlations vary modestly across populations. Future studies should assess the added values of MRCI, MPR, and PFR to population health management efforts.

J Manag Care Spec Pharm. 2020;26(7):860-71

Copyright $\odot 2020$, Academy of Managed Care Pharmacy. All rights reserved.

\section{What is already known about this subject}

Medication adherence and regimen complexity indices have been widely used in evaluating individual patient adherence at outpatient settings.

Given the increasing availability of observational health data, population-level medication adherence can be assessed using validated medication adherence indices.

\section{What this study adds}

Several barriers existed to automate the measurement of medication adherence indices from electronic health records (e.g., incompatible medication coding standards and data quality challenges)

Medication Regimen Complexity Index (MRCI) was modestly correlated with adherence measures such as medication possession ratio and prescription fill rates (Spearman correlation ranging from -0.17 to $-0.31, P<0.001$ ).

Distribution patterns of medication adherence indices were different in various subpopulations, with chronic patients having a higher MRCI (SD) compared with patients with no chronic conditions (16.9 [19.1] vs. 6.2 [6.6], $P<0.001)$.

$\mathrm{P}$ oor medication adherence is associated with prolonged treatments and adverse health outcomes. ${ }^{1,2}$ Low medication adherence incurs more than $\$ 290$ billion to the U.S. health system annually and considerably increases emergency room visits and hospitalizations on a population level. . $^{3-6}$ Failing to fill or refill a prescription, taking a lower dose than prescribed, and missing a dose are considered common medication nonadherence behaviors. ${ }^{7}$ Other contributing factors include undesired side effects, low levels of health care continuity, and contextual socioeconomic factors. ${ }^{7}$

Besides the patient behavior and socioeconomic context, complex medication regimens commonly result in poor adherence. ${ }^{8}$ Patients with chronic conditions often report difficulty in managing their medications, since instructions for multiple prescriptions need to be remembered, and some medications are challenging to use. ${ }^{9}$ Patients usually feel overwhelmed by prescription details, especially when physicians shift medications between different treatment courses. ${ }^{10}$ Thus, complex medication regimens are often considered a strong predictor of nonadherence that can potentially lead to higher health care utilization. ${ }^{11}$

Medication adherence is a multifaceted concept that can be measured using a variety of data sources such as surveys, administrative claims, and electronic health records (EHRs). Surveys are often used to collect self-reported individual-level 
behaviors; however, the validity and the generalizability of survey-derived adherence indices are usually confined by a patient's health literacy level, variety of evaluation scales, and small study sample sizes. ${ }^{12-14}$ Medication adherence surveys are also impractical to be administered at the point-of-care for all patients and visits. Insurers commonly use retrospective prescription claims data to measure adherence levels for each enrollee. For example, medication possession ratio (MPR), defined as the amount of medication furnished to a patient based on days supply and the number of days a patient should consume the medication, is an adherence index routinely chosen to classify patient adherence levels in commercial claims. ${ }^{15-17}$

Given the difficulty in administering surveys for all patients and challenges to collect insurance claims from various payers, health systems are increasingly using EHR data as an alternative source to measure population-level medication adherence. ${ }^{11}$ EHR-derived medication adherence indices enable health care providers to discern at-risk nonadhering patients based on previous encounters and explore interventions to better manage their patient populations.18,19 For example, the medication regimen complexity index (MRCI) quantifies medication management difficulty for patients and can be automatically calculated for all patients within an EHR. ${ }^{20,21}$ The prescription fill rate (PFR) is another index that captures the proportion of prescriptions filled by a patient; however, providers need both claims and EHR data to calculate it. ${ }^{11}$

Comparing various medication complexity and adherence indices is a growing need. Health systems operating within value-based boundaries (e.g., accountable care organizations) are increasingly using all types of clinical data, including EHRs and claims, for population health management purposes. ${ }^{22-29}$ However, only a few studies have investigated EHR-derived MRCI against claims-derived MPR, and none have offered a population-level scope. ${ }^{30-32}$ Furthermore, no attempt has been made to compare all 3 indices of MRCI, MPR, and PFR in a patient population of a health system. Comparing these measures enables health systems to identify patients experiencing difficulty with medication adherence and managing them more effectively with tailored interventions. ${ }^{11,33}$ We sought to develop a methodology to automate the generation of these indices due to data quality challenges of insurance claims and EHR data. ${ }^{34,35}$

In this study, we aimed to (a) describe multiple medication indices extracted from EHR and/or claims; (b) demonstrate the distribution of the medication-derived indices on a population level; and (c) assess the correlation between medication complexity and adherence indices.

\section{Methods}

\section{Data Source}

Data was provided by HealthPartners (Bloomington, MN), which is an integrated health care provider and health insurance company covering more than 1.5 million members. HealthPartners serves patients at 23 urgent care clinics and 27 in-clinic pharmacies at 55 locations and 7 hospitals throughout the Twin Cities (a major metropolitan area around MinneapolisSaint Paul, MN) and western Wisconsin. HealthPartners has adopted a centralized EHR system across all hospitals and outpatient facilities since $2008 .^{36}$

This study included outpatient structured EHR data medical claims, and pharmacy claims linked through unique patient identifiers. The claims data captured all health care services, including those rendered by providers outside of HealthPartners, billed to the patient medical insurance. EHR data of clinical encounters occurring outside of HealthPartners' network were not encompassed due to the lack of EHR interoperability with other health systems. ${ }^{22}$

Patient demographic characteristics, diagnostic codes (International Classification of Diseases, Ninth Revision, Clinical Modification [ICD-9-CM]), and medication codes (National Drug Code [NDC] numbers and medication generic internal ID) were extracted from EHRs and claims. EHR medication prescription data (i.e., prescription date, dosing frequency, dosing form, and additional medication instructions) were used to calculate MRCI..$^{20,21}$ Prescription fill dates and days of supply (from claims) were collected to calculate MPR. Medication prescribed dates (from EHRs), in addition to MPR data elements, were used to generate PFR. ${ }^{11}$

\section{Study Population Selection}

The original population included 114,665 patients in EHRs and 97,575 patients in claims. We identified those who enrolled in the HealthPartners insurance plan from 2012 to 2013 in the claims database and extracted the same cohort of patients from the EHR. We further excluded patients who (a) had data quality issues (280 patients in EHRs and 24 patients in claims); (b) were older than 65 years due to missing Medicare claims data (4,063 in EHRs and 3,761 in claims); (c) had no outpatient visit in 2012 or 2013 (14,004 in EHRs and 14,348 in claims); and (d) had no medication prescription records in EHRs (24,635 patients) or filled prescription records in claims (1,622 patients). The final study sample of eligible patients, with EHR and claims data for analysis, contained 70,054 individuals (Appendix A, available in online article).

\section{Medication Indices Construction}

Medication indices of MRCI, MPR, and PFR were constructed for 2012 and 2013. The patients eligible for inclusion might have multiple visits (include inpatient encounters) during the study period. To measure the medication complexity and 
adherence at outpatient-based physician settings, we excluded patients' medication records with flags of hospitalization (i.e., medications administered in an inpatient stay) to avoid the overcalculation from inpatient regimens, since medication adherence indices were initially designed for outpatient settings, ${ }^{8,20}$

Medication Regimen Complexity Index. MRCI was constructed based on an algorithm developed and validated in 2003. ${ }^{20}$ This instrument was further adapted for EHRs. ${ }^{21}$ MRCI is composed of 3 weighted elements: dosage form, dosing frequency, and additional administration instructions. We first calculated MRCI at the record level. We manually assigned weights to dosage forms (e.g., capsule, cream, or kit) in accordance to the routes of administration (e.g., oral, topical, and ophthalmic). ${ }^{20}$ Elements of dosage frequency and administration instructions were combined in a single data element as a semistructured format in the EHR. We broke down the text to MRCI components and assigned weights accordingly. ${ }^{20,37}$ We also confirmed the weights with a pharmacologist and a research specialist. Depending on the number of drugs that patients were prescribed, the MRCI of an individual can range from 1.5 (e.g., a single drug in tablet format to take once a day) to over 100 (depending on the frequency and mix of the medications). ${ }^{21}$ Two types of patientlevel MRCIs were collapsed based on 2 medication coding standards: the EHR's internal medication generic IDs and NDC numbers. This study reports 2012 EHR generic ID-based MRCIs. Results of the 2013 EHR generic ID-based MRCI and NDC-based MRCIs of both years are included in Appendix B (available in online article).

Medication Possession Ratio. Using claims only, the numerator for MPR was calculated by summing the days supply from the first to the last prescription (including the last supply), while the denominator was the time between the first and last prescription dates and the last days supply. MPR applies to cases in which the prescriptions have been filled for more than 1 time (i.e., had a refill).

Proportion of days covered (PDC) is another measure recommended for assessing the medication adherence of patients on multiple therapies. MPR and PDC are very similar measures by definition; however, because of data limitations, calculating PDC was more challenging and prone to errors compared with MPR. For example, identifying drug switching or the usage of dual therapy in patient pharmacy claims is a complicated task that can lead to underestimating PDC. Therefore, we only measured MPR as a refill adherence index. For cases of multiple medications, MPR was calculated by the days supply averaged over all drugs divided by the days between the first prescription date and last prescription date (inclusive). MPR ranged from $0 \%$ to $100 \%$ but could also result in values more than $100 \% .{ }^{38} \mathrm{MPR} \geq 80.0 \%$ is the cutoff considered to be good medication adherence, and MPR $<50.0 \%$ indicates poor adherence. ${ }^{39}$

In order to capture the prescription fills with multiple drug products, which contain similar ingredients, we mapped claims-extracted NDC numbers to drug active ingredient categories. MPR was constructed based on active ingredient categories and NDC numbers for 2012 and 2013. This study reports MPR indices derived from active ingredients in 2012 Other years and versions of MPRs are reported in Appendix B.

Prescription Fill Rate. PFR is a patient-level indicator referring to the proportion of prescriptions filled within a designated period (7 days or 30 days). ${ }^{11}$ PFR reflects a patient's intention to adhere to the medication orders following an encounter. Linked patient-level EHR and claims data are required to calculate PFRs because of potential variations between prescription NDC numbers and filled NDC numbers (e.g., pharmacists may dispense slightly different packages of a medication for the same prescription). ${ }^{11}$ We adopted the Johns Hopkins Adjusted Clinical Group (ACG) system (version 11) to convert NDC numbers into 1 of 62 prescribed medication-defined morbidity groups (RxMGs). ${ }^{40}$ Each NDC number was mapped to 1 RxMG category based on the active ingredient, route of administration, intended therapeutic use, and the mechanisms of action of that medication. ${ }^{40}$ The ACG system is a validated risk stratification software program that categorizes underlying data types into broader coding groups. ${ }^{40}$ By matching RxMGs from EHRs to claims for each patient, we calculated the proportion of prescribed RxMGs filled within 7 days and 30 days from the prescription date. ${ }^{11}$

Other Indices. To assess the association of a patient's morbidity, prescription history, and medication adherence, we calculated several measures at the individual level. Unique ICD (5-digit subcategories) and NDC counts (i.e., prescription count in EHRs and filled prescription count in claims) were calculated. Patient comorbidity levels were given by a score using the Charlson Comorbidity Index algorithm mapping to 17 chronic conditions in the claims data. ${ }^{41}$ Total patient count of chronic conditions was calculated using ICD-9-CM claim codes grouped by Agency for Healthcare and Research Quality's Chronic Condition Indicator. ${ }^{42,43}$

\section{Statistical Analysis}

We described the population demographic characteristics (e.g., age, sex, marital status, and language); health care utilization (e.g., outpatient visits, inpatient visits, emergency department [ED] visits, and unplanned readmissions); and 3 medication indices for the entire study sample. We then stratified the results by age, sex, chronic conditions, and inpatient visit status. Analysis of variance, t-tests, and Kruskal-Wallis tests were conducted to examine the variation of medication indices among subpopulations. We generated the overlapping density 


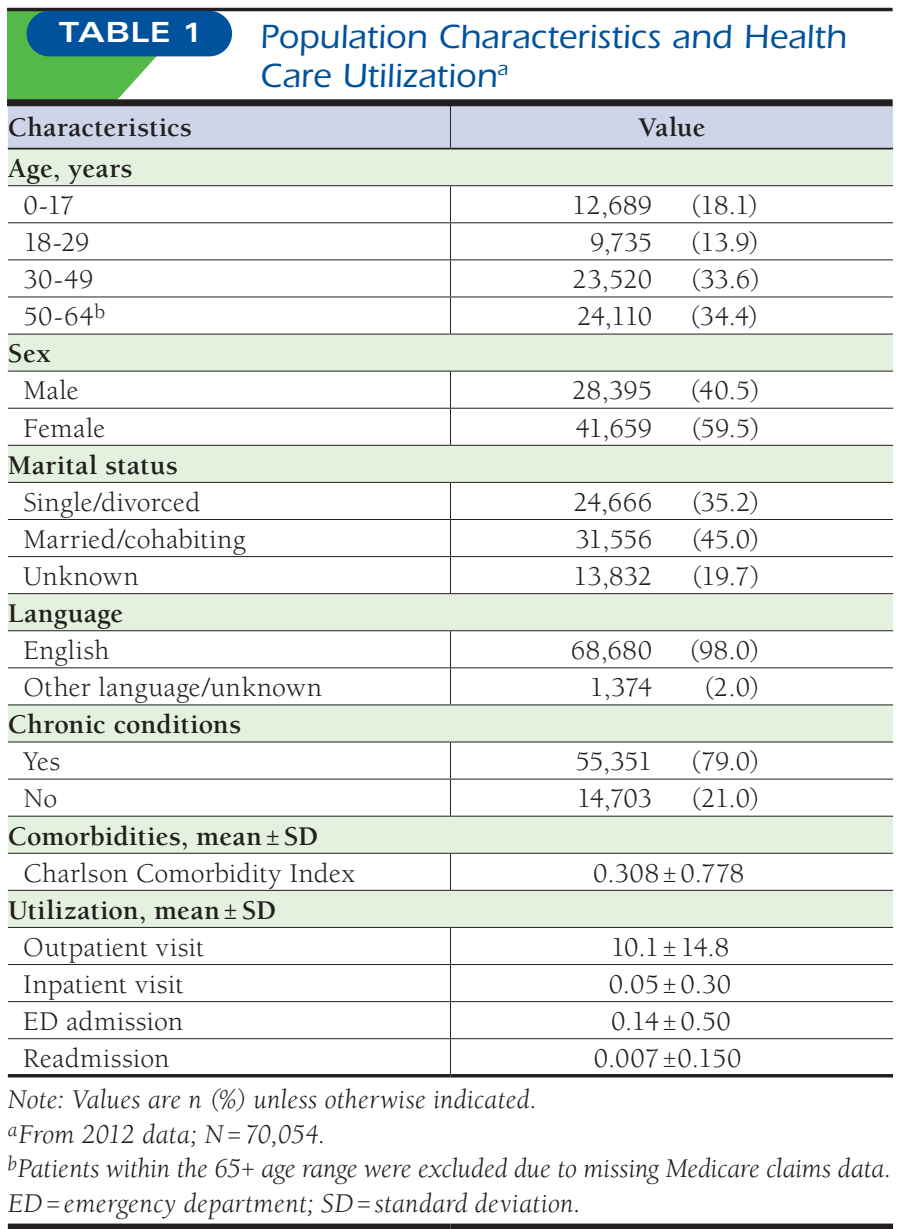

plot of 30-day PFR, MPR, MRCI and prescription counts (EHR) to compare the indices on a population level. Partial correlation with Spearman coefficient (SC) was estimated between indices adjusted for age and sex.

Sensitivity analysis was performed by further adjusting for chronic conditions and hospitalization in the general population and evaluating indices correlation in a subpopulation with chronic conditions. Partial correlation analysis results with Pearson's coefficient were also assessed by sensitivity analysis. We conducted nonparametric tests by bootstrapping the indices to measure the significant level of correlation (i.e., $P$ value). Statistical analyses were conducted using $\mathrm{R}$, version 3.5.1 (Foundation for Statistical Computing, Vienna, Austria).

\section{Results}

\section{Population Characteristics}

Mean (standard deviation [SD]) age of the study population was 37.9 (18.0) years, and more than half (59.5\%) of the patients were females (Table 1). About $79.0 \%$ of patients had a chronic condition, and the mean value (SD) of Charlson Comorbidity

\section{TABLE 2 Medication Complexity and Adherence} Indices

\begin{tabular}{|c|c|c|}
\hline Medication Indices ${ }^{a}$ & EHR & Claims \\
\hline $\mathrm{MRCI}(\text { mean } \pm \mathrm{SD})^{\mathrm{b}}$ & $14.6 \pm 17.8$ & - \\
\hline ICD count $($ mean \pm SD $)$ & $14.3 \pm 16.6$ & $13.4 \pm 9.7$ \\
\hline$<10$ & $35,070 \quad(50.1)$ & $28,765 \quad(41.1)$ \\
\hline $10-19$ & $19,321 \quad(27.6)$ & $27,849 \quad(39.8)$ \\
\hline $20-39$ & $10,960 \quad(15.6)$ & $11,682 \quad(16.7)$ \\
\hline$>40$ & $4,222 \quad(6.0)$ & $1,548 \quad(2.2)$ \\
\hline Missing & 481 & 210 \\
\hline Prescription count $($ mean \pm SD) & $4.0 \pm 3.7$ & $5.5 \pm 5.1$ \\
\hline$<3$ & $34,658 \quad(49.4)$ & $25,067 \quad(36.5)$ \\
\hline $3-10$ & $30,596 \quad(43.7)$ & $34,587 \quad(49.4)$ \\
\hline$>10$ & $4,800 \quad(6.9)$ & $9,860 \quad(14.1)$ \\
\hline MPR $(\text { mean } \pm \text { SD }, \%)^{b}$ & - & $62.4 \pm 31.0$ \\
\hline$>80.0$ & - & $15,105 \quad(21.6)$ \\
\hline $50.0-80.0$ & - & $15,838 \quad(22.6)$ \\
\hline$<50.0$ & - & $16,674 \quad(23.8)$ \\
\hline NA & - & $22,437 \quad(32.0)$ \\
\hline 7-day PFR $($ mean \pm SD,$\%)$ & $71.5 \pm 31.9$ & \\
\hline 30-day PFR (mean \pm SD, \%) & $81.0 \pm 27.0$ & \\
\hline
\end{tabular}

Note: Values are $n(\%)$ unless otherwise indicated.

aPopulation characteristics and medication records were derived from 2012 data; $N=70,054$.

${ }^{b}$ MRCI was developed based on EHR's internal generic ID; MPR was developed based on active ingredients of medications.

$E H R=$ electronic health record; ICD = International Classification of Diseases; $M P R=$ medication possession ratio; $M R C I=$ medication regimen complexity index $N A=$ not available; $P F R=$ prescription fill rate; $S D=$ standard deviation

Index scores was 0.308 (0.778). On average, patients in this population had 10.1 (14.8) outpatient visits, $0.05(0.30)$ inpatient visits, 0.14 (0.50) ED admissions, and $0.007(0.150)$ unplanned readmissions in 2012 (Table 1).

\section{Medication Complexity and Adherence Indices}

Mean (SD) prescription count of EHR was 4.0 (3.7), generating a mean (SD) MRCI of 14.6 (17.8; Table 2). Average (SD) MPR was 62.4 (31.0) among those who had refill records in 2012, but $32.0 \%$ of our study sample did not have any refills so were missing an MPR index. Close to 22\% of patients adhered well to their outpatient medications (MPR $\geq 80.0 \%$ ), while about $24 \%$ showed poor adherence (MPR $<50.0 \%)$. The 7-day and 30-day PFRs (SD) were 71.5 (31.9)\% and 81.0 (27.0)\% (Table 2).

\section{Distribution and Stratification of Medication Indices}

Most patients were not severely ill, only receiving a few prescriptions, and the regimens for them were not complex to manage (i.e., Medicare data were inaccessible, so older adults were excluded). Thus, the distributions of EHR prescription count and MRCI were right skewed. Density plots showed that EHR prescription count and MRCI followed similar distribution pattern (Figure 1). However, the shapes of 30-days PFR and MPR density conveyed different information for 


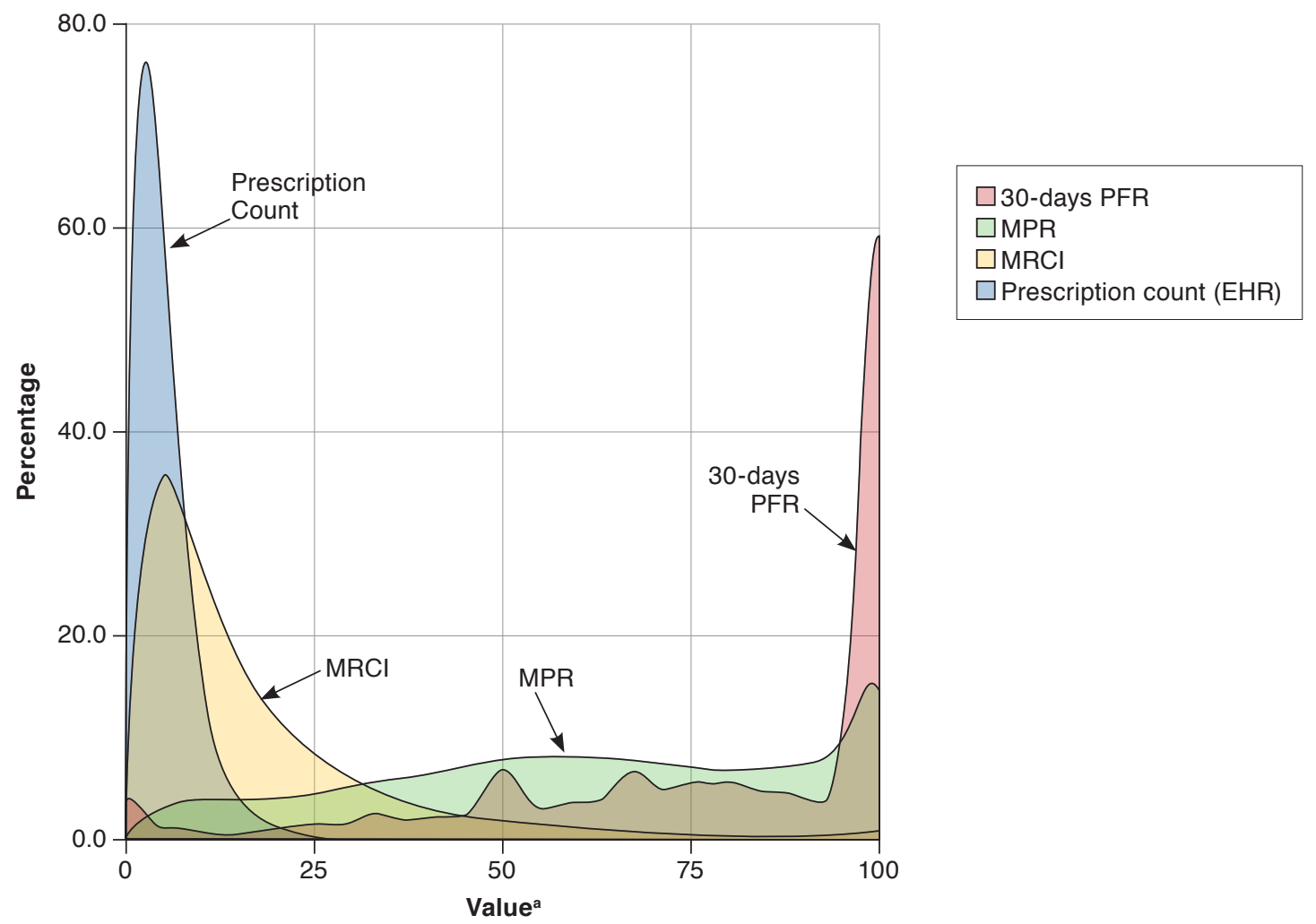

aValue in x-axis represents numeric value for MRCI and prescription count, whereas percentages are used for 30-days PFR and MPR.

$E H R=$ electronic health record; $I C D=$ International Classification of Diseases; $M P R=$ medication possession ratio; $M R C I=$ medication regimen complexity index; $P F R=$ prescription fill rate.

primary fill and refill behaviors (Figure 1). About $16.0 \%$ of the population were identified to have nearly perfect adherence to their prescriptions (MPR $\geq 95.0 \%$ ). PFR results indicated that around half of patients from the total population filled all their prescriptions in 7 days (45.2\%) and slightly more when the time was extended to 30 days (54.6\%). Except for the population with perfect adherence levels, the rest of patients did not demonstrate any significant trends in primary filling or refilling (Figure 1).

The mean Charlson Comorbidity Index score (SD) for males was higher than for females (0.332 [0.817] vs. 0.292 [0.751], $P<0.001$ ); however, the MRCI (SD) (13.7 [16.4] vs. 15.3 [18.6], $P<0.001)$ for males was lower than for females. There was no significant difference in MPR (SD; $62.0[32.6] \%$ vs. 62.6 [30.1]\%, $P>0.05$ ) between genders (male vs. female). Medication prescription count and MRCI increased by age (from $<18$ years to 50-64 years). Patients with an inpatient stay were likely to have had high MRCIs, despite excluding inpatient medications from MRCI calculations (33.2 [33.0] vs. 13.9 [16.4], P<0.001), so as patients with chronic conditions compared with no chronic conditions (16.9 [19.1] vs. 6.2 [6.6], $P<0.001$ ). Patients with an inpatient stay were less adherent to their outpatient prescriptions $(\mathrm{MPR}=50.3[27.6] \%$ vs. 63.0 [31.1]\%, $P<0.001$ ), but adherence was significantly higher among patients with chronic conditions than without chronic conditions (MPR $=62.6[29.7] \%$ vs. 60.5 [39.2]\%, $P<0.001$; Table 3).

\section{Partial Correlation of Indices}

Medication adherence measures of MPR and PFR were negatively correlated with MRCI, ICD counts, and prescription NDC counts. The correlation improved slightly after adjusting for population age and sex (Figure 2). Partial correlation between MRCI and 30-day PFR was also minimal ( $S C=-0.17$, $P<0.001$; Figure 2). The correlation between MPR and MRCI $(S C=-0.31, P<0.001$; Figure 2) was slightly lower than that between MPR and claims-derived prescription count $(S C=-0.37, P<0.001)$. The partial correlation between MPR and MRCI in patients with chronic conditions gave close 


\begin{tabular}{|c|c|c|c|c|c|c|}
\hline & \multicolumn{3}{|c|}{ EHRs } & \multicolumn{2}{|c|}{ Claims } & \multirow{2}{*}{$\begin{array}{c}\text { Both } \\
\text { PFR (30 days, \%) }\end{array}$} \\
\hline & ICD Count & Rx Count & MRCI & MPR (\%) & $\begin{array}{c}\text { Charlson } \\
\text { Comorbidity Index } \\
\end{array}$ & \\
\hline \multicolumn{7}{|c|}{ Age, years $(\text { mean } \pm S D)^{a}$} \\
\hline$<18$ & $10.3 \pm 11.4$ & $2.6 \pm 2.1$ & $10.8 \pm 12.6$ & $46.9 \pm 35.9$ & $0.151 \pm 0.404$ & $89.2 \pm 23.7$ \\
\hline $18-29$ & $10.8 \pm 13.8$ & $3.3 \pm 2.9$ & $11.66 \pm 14.0$ & $66.0 \pm 34.1$ & $0.152 \pm 0.448$ & $79.6 \pm 28.7$ \\
\hline $30-49$ & $13.6 \pm 19.8$ & $3.9 \pm 4.3$ & $14.1 \pm 20.9$ & $62.1 \pm 26.1$ & $0.253 \pm 1.029$ & $80.2 \pm 26.4$ \\
\hline $50-64$ & $18.6 \pm 15.4$ & $5.1 \pm 3.5$ & $18.5 \pm 17.3$ & $65.7 \pm 32.0$ & $0.506 \pm 0.694$ & $78.8 \pm 27.7$ \\
\hline \multicolumn{7}{|c|}{ Sex $(\text { mean } \pm \text { SD) })^{a}$} \\
\hline Male & $12.5 \pm 14.7$ & $3.7 \pm 3.2$ & $13.7 \pm 16.4$ & $62.0 \pm 32.6^{b}$ & $0.332 \pm 0.817$ & $82.7 \pm 26.5$ \\
\hline Female & $15.6 \pm 17.7$ & $4.3 \pm 3.9$ & $15.3 \pm 18.6$ & $62.6 \pm 30.1$ & $0.292 \pm 0.751$ & $80.0 \pm 27.2$ \\
\hline \multicolumn{7}{|c|}{ Chronic condition (mean \pm SD) ${ }^{a}$} \\
\hline No & $6.4 \pm 6.4$ & $2.0 \pm 1.3$ & $6.2 \pm 6.6$ & $60.5 \pm 39.2$ & $0.053 \pm 0.302$ & $87.5 \pm 26.6$ \\
\hline Yes & $16.4 \pm 17.8$ & $4.5 \pm 3.9$ & $16.9 \pm 19.1$ & $62.6 \pm 29.7$ & $0.375 \pm .849$ & $79.7 \pm 26.9$ \\
\hline \multicolumn{7}{|c|}{ Inpatient visit $\left(\right.$ mean \pm SD) ${ }^{a}$} \\
\hline No & $13.4 \pm 14.4$ & $3.9 \pm 3.4$ & $13.9 \pm 16.4$ & $63.0 \pm 31.1$ & $0.271 \pm 0.691$ & $81.3 \pm 27.1$ \\
\hline Yes & $36.3 \pm 37.9$ & $8.0 \pm 6.4$ & $33.2 \pm 33.0$ & $50.3 \pm 27.6$ & $1.204 \pm 1.696$ & $75.7 \pm 23.6$ \\
\hline \multicolumn{7}{|c|}{$\begin{array}{l}\text { a Analysis of variance or t-tests were performed to evaluate the difference of variance for ICD count, prescription count, MRCI, and Charlson Comorbidity Inc } \\
\text { subcategories. The nonparametric Kruskal-Wallis test was performed for MPR and PFR. } \\
\text { bAll within-group comparisons had significantly different medication indices at } P<0.001 \text { except MPR in male and female patients. } \\
\text { ICD = International Classification of Diseases; MPR = medication possession ratio; MRCI= medication regimen complexity index; PFR=prescription fill rate; } \\
R x=\text { prescription; } S D=\text { standard deviation. }\end{array}$} \\
\hline
\end{tabular}

values. Correlation analyses results of paired indices remained consistent in 2012 and 2013, so as in the sensitivity analysis, which were adjusted for chronic condition and hospitalization. Results of the correlation analysis using Pearson's coefficient also showed a similar pattern.

\section{Discussion}

Health care providers are increasingly using EHR data, along with insurance claims, to improve the management of their patient populations. ${ }^{22-29}$ Medication complexity and adherence indices can boost such efforts by providing clinicians and case managers with key information to improve treatment outcomes and reduce utilization. ${ }^{11}$ In this study, we reviewed medication data from a 2-year retrospective cohort of 70,054 patients with linked claims and EHR data provided by an integrated health care delivery network. We reconstructed 3 major medication indices, MRCI, MPR and PFR, to evaluate medication regimen management complexity and medication adherence at the population level. We found the distribution of these derived measures differentiated when stratified by population demographic characteristics or hospital utilization. The MRCI closely followed the distribution of EHR prescription count. A large number of patients adhered to their regimens (only $23.8 \%$ of patients had an MPR $<50 \%$; Table 1 ), whereas the distribution of MPR and PFR did not demonstrate a clear pattern (Figure 1). MPR and PFR indices were in loose correlation with MRCI (Figure 2).
Our analysis showed that the correlation between MRCI and MPR, or MRCI and PFR, were not very significant. Several reasons could possibly account for these observations. First, we attempted to replicate a real-world scenario in which providers of a health system have access to their own EHR data and not total EHR records of all patients across all providers in the United States (i.e., inherent EHR data leakage from one provider to another); however, health plans have access to claims data of patient encounters across all providers. Thus, our results may be an indication of the underlying data limitations in the real world, thus, providing insight on what type of an adherence measure a health care provider network can calculate versus a health plan. Moreover, assuming that EHRs were missing at random versus their claim records, the correlation outcomes should be generalizable to health care networks that have EHRs with minimal leakage (e.g., staff-modeled health maintenance organizations).

Second, the correlations can be explained by the fact that MPR and PFR only reflect patient nonadherence behaviors at certain dimensions. MPR demonstrates the medication nonadherence in a few perspectives (e.g., delay refills, non-refill or skip fill for once) but is incapable of capturing other nonadherence behaviors, such as skipping the doses, splitting pills, or stopping medications early. ${ }^{7}$ Similarly, PFR only measures the patient's compliance at the primary fill (i.e., whether the prescription was filled in time during a given period). 


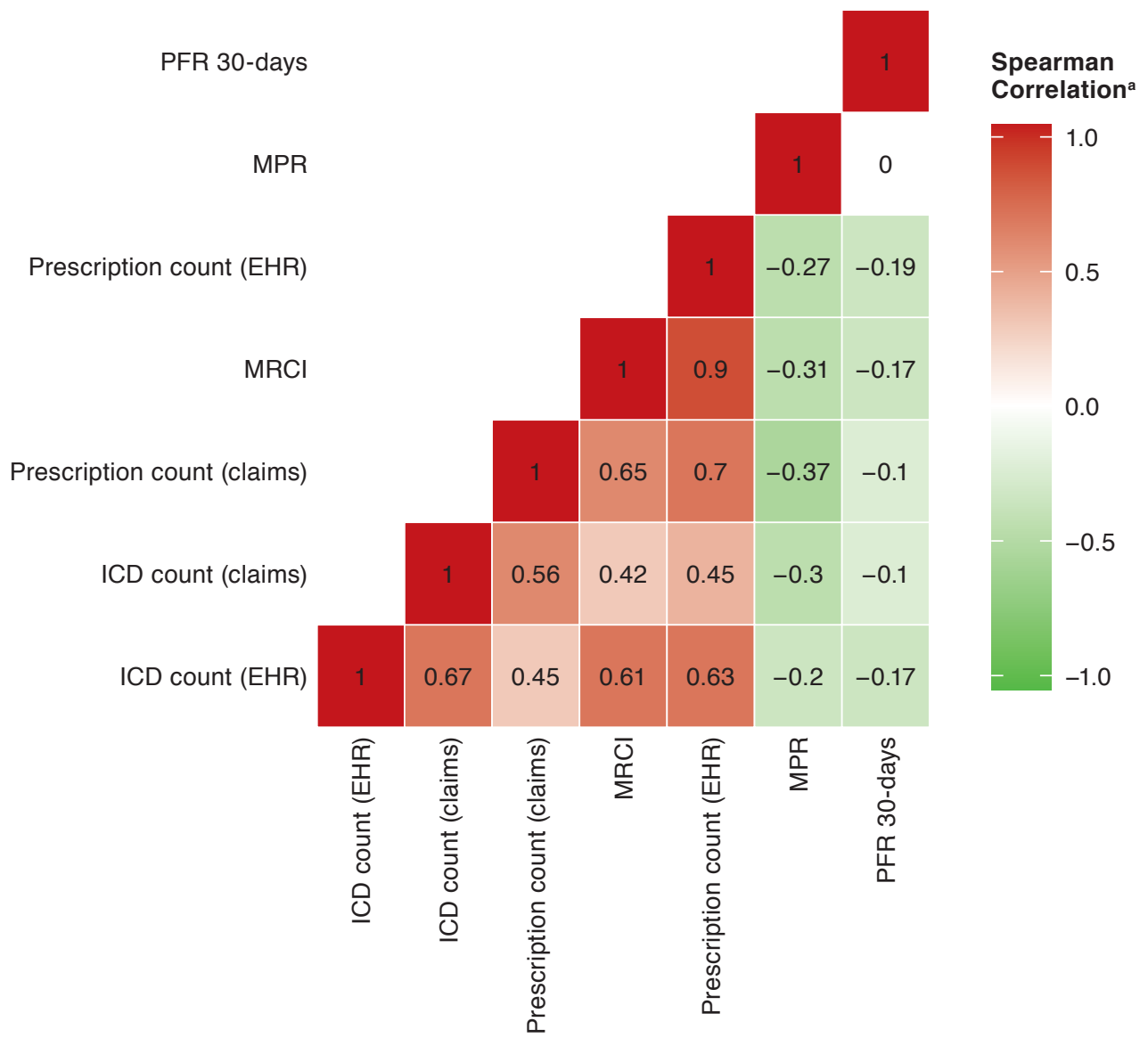

apartial correlation was calculated after adjusting for age and sex.

$E H R=$ electronic health record; ICD = International Classification of Diseases; $M P R=$ medication possession ratio; $M R C I=$ medication regimen complexity index; $P F R=$ prescription fill rate

Third, nonadherence was not only a result of complex medication regimens. ${ }^{44}$ Poor physician-patient communication, patient dissatisfaction to treatment plan, ${ }^{45}$ and other health systems and socioeconomic factors are also acknowledged as common barriers to effective medication use. ${ }^{46}$ In this sense, the adherence was not well represented by the individual measures we used. The MRCI also only accounted for a specific dimension of nonadherence (i.e., medication complexity).

Finally, certain correlates of medication adherence, such as detailed disease severity levels, are often not measurable using the EHR or claims data. Therefore, our correlation results should be interpreted within the limitations of real-world data (e.g., EHR data leakage), as well as limitations of adherence indices used in this study (e.g., medication complexity is only one aspect of adherence). Future studies should enhance available adherence instruments or tailor new ones that perform well using existing structured patient data at large volume (e.g., produce higher correlations regardless of the clinical data choice).

We faced several barriers when automating the extraction of medication records and development of adherence measures using the EHR and claims data. Logically, the procedure to generate MRCI, MPR, and PFR indices starts with extracting medication records and then grouping the medication-level indices to the patient level. However, because of the complexity of mapping multiple medication coding systems, matching the exact prescribed and filled products across databases was not always consistent. For example, medication coding standards may shift as patient information is passed from an EHR to a claims database. ${ }^{47}$ Besides, pharmacies may substitute the prescribed products due to lack of inventory, which introduces additional variation in patient pharmacy claims. 
In this study, we assessed several distinctive NDC-derived medication clusters to develop MRCI, MPR, and PFR indices. As a result, although the actual values of the indices changed when composing with different NDC groupers (e.g., ACG's RxMG), the distribution pattern and correlation with other indices held the same trends (Appendix B). However, the variations in prescription coding versus filling codes may become problematic when comparing the plain value of indices from one study to another. Additionally, the elements required for specific MRCI components are often stored in semistructured formats in EHRs, adding yet another layer of complexity to generalize the methodology across different health systems. Therefore, future studies need to be cautious when automating MRCI, MPR, and PFR measures using EHR and claims data across large denominators of patients.

Clinical providers are increasingly using EHR data for population health management purposes. ${ }^{22-23,48-53}$ We observed apparent unconformity in MRCI, MPR, and PFR distribution when stratifying the population by its utilization history, which implied that these novel medication indices could probably add to existing EHR-derived utilization risk stratification models. ${ }^{22-26}$ Some studies had considered integrating MRCI, ${ }^{54-57}$ MPR, ${ }^{58-61}$ or $\mathrm{PFR}^{11}$ into predictive models for medical cost, pharmacy cost, or hospital utilization forecasting, but these studies commonly focused on particular conditions or small sample sizes.

Based on our preliminary results, risk adjustment models may benefit from simple and complex medication-derived indices in predicting health care utilization. However, it needs to be emphasized that the construction of these medication indices requires detailed notes on patient medications, which is not usually the case for the general population, except for highly comorbid patients. For example, information for a single patient may be available to construct PFR, but probably would not be adequate to calculate MRCI. The fairness of parallel comparison for medication indicators should be taken into account when developing predictive models. Much effort is required to understand the value of prescription data extracted from EHRs in forecasting utilization.

\section{Limitations}

This study has some limitations to consider. First, as an integrated high-performing delivery system, HealthPartners provides most of its patient care services within the delivery network; however, we still missed the medical records administrated by other health care organizations but not shared with HealthPartners. We did not evaluate the proportion of encounter data leaking from the HealthPartners' EHR or identified those with limited activity within the health care network. The correlation measurements might lose their robustness when more data sources can be linked for further analysis. Also, we lacked the fill information of out-of-pocket, over-the-counter, and sample drugs (i.e., often missing in claims). ${ }^{11}$ Hence, the complexity of medication management might have been underestimated.

Second, there have been a handful of studies validating the medication-derived measures in pediatric patients, ${ }^{62-65}$ although children's adherence to medication could be affected by more complex factors such as family trust and caregiver involvement. The correlation assessment should be validated and needs further investigation in the pediatric population.

Third, the population included in this study was limited to commercially insured patients, since we were unable to trace the drug usage and adherence behaviors of those aged 65 years and older (i.e., Medicare claims were not accessible). Older adults usually have a higher complexity of medication regimens, which can result in a different pattern of and correlation between MRCI and MPR or PFR.

Finally, we did not investigate the temporal changes of medication complexity and adherence indices. An entire year can be too long or too short to assess patient adherence. For example, in long-term chronic conditions, the effect of medication complexity may take multiple years to show its effect on adherence, while for acute conditions the effect may washout within days or weeks.

A prominent challenge was the lack of well-acknowledged guidelines documenting MRCI and MPR construction for population health data. Selecting medication coding standard remained a problem in the process of automation. It was technically challenging to convert NDC numbers (widely used in administrative health databases) to other standardized medication codes (e.g., RxNorm) in order to address drug product duplication issues. Using other hierarchical clusters may lose detailed information from patients' medication records. Besides, partial prescription data were not well structured in EHR; hence, current procedures for developing MRCI based on EHR data inevitably depends on a manual process supervised by a pharmacist. Future studies should explore the application of basic text mining techniques to facilitate components segmentation, thereby expediting the calculation of EHR-derived MRCI.

\section{Conclusions}

Medication adherence differed by population demographic features and hospital utilization. Regimen complexity affects patient adherence to medication, but the correlation was not significant as represented by medication indices derived from insurance claims. Future studies should explore the development of measures to quantify additional medication compliance behaviors, as well as using medication complexity and adherence indices in risk stratification models of health care utilization. 


\section{Authors}

XIAOMENG MA, MS; HSIEN-YEN CHANG, PhD; and THOMAS M. RICHARDS, MS, Center for Population Health IT, Department of Health Policy and Management, Johns Hopkins Bloomberg School of Public Health, Baltimore, Maryland. CHANGMI JUNG, PhD, Carey Business School, Johns Hopkins University, Baltimore, Maryland. HADI KHARRAZI, MD, PhD, Center for Population Health IT, Department of Health Policy and Management, Johns Hopkins Bloomberg School of Public Health, and Division of Health Sciences and Informatics, Johns Hopkins School of Medicine, Baltimore, Maryland.

AUTHOR CORRESPONDENCE: Hadi Kharrazi, MD, PhD, Research Director \& Associate Professor, 624 N. Broadway, \#606, Baltimore, MD 21205. Tel.: 443.287.8264; E-mail: kharrazi@jhu.edu.

\section{DISCLOSURES}

No outside funding supported this study. The authors have nothing to disclose.

The abstract of this work was presented at INFORMS Healthcare Conference, held on July 27-29, 2019, in Cambridge, MA.

\section{ACKNOWLEDGMENT}

We acknowledge the support of HealthPartners (Bloomington, MN) in sharing the underlying data and providing the research team with technical support throughout the research.

\section{REFERENCES}

1. Hsu KL, Fink JC, Ginsberg JS, et al. Self-reported medication adherence and adverse patient safety events in CKD. Am J Kidney Dis. 2015;66(4):621-29.

2. Li L, Cui Y, Yin R, et al. Medication adherence has an impact on disease activity in rheumatoid arthritis: a systematic review and meta-analysis. Patient Prefer Adherence. 2017;11:1343-56.

3. Langer Research Associations. Medication adherence in America: a national report 2013. National Community Pharmacists Association. Available at: http://www.ncpa.co/adherence/AdherenceReportCard_Full.pdf. Accessed June 1, 2019.

4. Iuga AO, McGuire MJ. Adherence and health care costs. Risk Manag Healthc Policy. 2014;7:35-44.

5. Sokol MC, McGuigan KA, Verbrugge RR, et al. Impact of medication adherence on hospitalization risk and healthcare cost. Med Care. 2005; 43(6)521-30.

6. Lo-Ciganic WH, Donohue JM, Jones BL, et al. Trajectories of diabetes medication adherence and hospitalization risk: a retrospective cohort study in a large state Medicaid program. J Gen Intern Med. 2016;31(9):1052-60.

7. Sabaté E. Adherence to long-term therapies: evidence for action. World Health Organization. 2003. Available at: https://www.who.int/chp/ knowledge/publications/adherence_full_report.pdf. Accessed June 5, 2020.

8. Osterberg L, Blaschke T. Adherence to medication. N Engl J Med. 2005;353(5):487-97.

9. Mishra SI, Gioia D, Childress S, et al. Adherence to medication regimens among low-income patients with multiple comorbid chronic conditions. Health Soc Work. 2011;36(4):249-58

10. Bosworth HB, Granger BB, Mendys P, et al. Medication adherence: a call for action. Am Heart J. 2011;162(3):412-24.
11. Chang HY, Richards TM, Shermock KM, et al. Evaluating the impact of prescription fill rates on risk stratification model performance. Med Care. 2017;55(12):1052-60.

12. Nguyen TM, Caze AL, Cottrell N. What are validated self-report adherence scales really measuring? A systematic review. Br J of Clin Pharmacol. 2014;77(3):427-45.

13. Stirratt MJ, Dunbar-Jacob J, Crane HM, et al. Self-report measures of medication adherence behavior: recommendations on optimal use. Transl Behav Med. 2015;5(4):470-82

14. Basu S, Garg S, Sharma N, et al. Improving the assessment of medication adherence: challenges and considerations with a focus on low-resource settings. Ci Ji Yi Xue Za Zhi. 2019;31(2):73-80.

15. Briesacher BA, Andrade SE, Fouayzi H, et al. Comparison of drug adherence rates among patients with seven different medical conditions. Pharmacotherapy. 2008;28(4):437-43

16. Tang KL, Quan H, Rabi DM. Measuring medication adherence in patients with incident hypertension: a retrospective cohort study. BMC Health Serv Res. 2017;17(1):135

17. Leader J, Wood GC, Shah N, et al. PS3-03: Creation and validation of the electronic medication possession ratio (eMPR). Clin Med Res. 2010;8(3-4):187.

18. Fischer MA, Stedman MR, Lii J, et al. Primary medication non-adherence: analysis of 195,930 electronic prescriptions. J Gen Intern Med. 2010;25(4): 284-90.

19. Kirkman MS, Rowan-Martin MT, Levin R, et al. Determinants of adherence to diabetes medications: findings from a large pharmacy claims database. Diabetes Care. 2015;38(4):604-09.

20. George J, Phun YT, Bailey MJ, et al. Development and validation of the medication regimen complexity index. Ann Pharmacother. 2004;38(9): 1369-76

21. McDonald MV, Peng TR, Sridharan S, et al. Automating the medication regimen complexity index. J Am Med Inform Assoc. 2012;20(3):499-505.

22. Kharrazi H, Chi W, Chang HY, et al. Comparing population-based risk-stratification model performance using demographic, diagnosis and medication data extracted from outpatient electronic health records versus administrative claims. Med Care. 2017;55(8):789-96.

23. Kharrazi H, Weiner JP. A practical comparison between the predictive power of population-based risk stratification models using data from electronic health records versus administrative claims: setting a baseline for future EHR-derived risk stratification models. Med Care. 2018;56(2):202-03.

24. Kan HJ, Kharrazi H, Leff B, et al. Defining and assessing geriatric risk factors and associated health care utilization among older adults using claims and electronic health records. Med Care. 2018;56(3):233-39.

25. Lemke KW, Gudzune KA, Kharrazi H, Weiner JP. Assessing markers from ambulatory laboratory tests for predicting high-risk patients. Am J Manag Care. 2018;24(6):e190-95.

26. Kharrazi H, Chang HY, Heins SE, et al. Assessing the impact of body mass index information on the performance of risk adjustment models in predicting health care costs and utilization. Med Care. 2018;56(12):1042-50.

27. Kan HJ, Kharrazi H, Chang HY, Bodycombe D, Lemke K, Weiner JP. Exploring the use of machine learning for risk adjustment: a comparison of standard and penalized linear regression models in predicting health care costs in older adults. PLoS One. 2019;14(3):e0213258.

28. Hatef E, Searle KM, Predmore Z, et al. The impact of social determinants of health on hospitalization in the Veterans Health Administration. Am J Prev Med. 2019;56(6):811-18.

29. Hatef E, Kharrazi H, Searle KM, et al. The association between neighborhood socioeconomic and housing characteristics with hospitalization: results of a national study of veterans. J Am Board Fam Med. 2019;32(6):890-903. 
30. Ho CP, Yeh JI, Wen SH, et al. Associations among medication regimen complexity, medical specialty, and medication possession ratio in newly diagnosed hypertensive patients: a population-based study. Medicine (Baltimore). 2017;96(45):e8497.

31. Goldstein CM, Gathright EC, Gunstad J, et al. Depressive symptoms moderate the relationship between medication regimen complexity and objectively measured medication adherence in adults with heart failure. J Behav Med. 2017;40(4):602-11.

32. Ghimire S, Peterson GM, Castelino RL, et al. Medication regimen complexity and adherence in haemodialysis patients: an exploratory study. Am J Nephrol. 2016;43(5):318-24.

33. Alves-Conceicao V, Rocha KS, Silva FV, et al. Medication regimen complexity measured by MRCI: a systematic review to identify health outcomes. Ann Pharmacother. 2018;52(11):1117-34.

34. Lucy K, Tang K, Quan H. Barriers to data quality resulting from the process of coding health information to administrative data: a qualitative study. BMC Health Serv Res. 2017;17(1):766.

35. Kharrazi H, Wang C, Scharfstein D. Prospective EHR-based clinical trials: the challenge of missing data. J Gen Intern Med. 2014;29(7):976-78.

36. HealthPartners. Quick facts. Retrieved September 7, 2019. Available at: https://www.healthpartners.com/about/facts/. Accessed June 2, 2020.

37. Sheehan OC, Kharrazi H, Carl KJ, et al. Helping older adults improve their medication experience (home) by addressing medication regimen complexity in home healthcare. Home Healthc Now. 2018;36(1):10-19.

38. American Pharmacists Association. Measuring adherence. Retrieved September 7, 2019] Available at: https://www.pharmacist.com/measuringadherence. Accessed June 2, 2020.

39. Karve S, Cleves MA, Helm M, et al. Good and poor adherence: optimal cut-point for adherence measures using administrative claims data. Current Med Res Opin. 2009;25(9):2303-10.

40. The Johns Hopkins University Bloomberg School of Public Health, Health Services Research and Development Center. The Johns Hopkins ACG casemix system reference manual, version 11.0. Technical reference guide. 2014 Available at: https://www.hopkinsacg.org/resource-center/\#documentation. Accessed June 2, 2020.

41. Brusselaers N, Lagergren J. The Charlson Comorbidity Index in registrybased research. Methods Inf Med. 2017;56(05):401-06.

42. Healthcare Cost and Utilization Project (HCUP). Chronic Condition Indicator (CCI) for ICD-9-CM. May 2016. Available at: https://www.hcupus.ahrq.gov/toolssoftware/chronic/chronic.jsp\#files. Accessed June 2, 2020.

43. Chi M, Lee C, Wu S. The prevalence of chronic conditions and medical expenditures of the elderly by Chronic Condition Indicator (CCI). Arch Gerontol Geriatr. 2011;52(3):284-89.

44. Brysch EG, Cauthon KA, Kalich BA, et al. Medication regimen complexity index in the elderly in an outpatient setting: a literature review. Consult Pharm. 2018;33(9):484-96.

45. Jimmy B, Jose J. Patient medication adherence: measures in daily practice. Oman Med J. 2011;26(3):155-59.

46. Brown MT, Bussell JK. Medication adherence: WHO cares? Mayo Clin Proc. 2011;86(4):304-14.

47. Richesson RL. An informatics framework for the standardized collection and analysis of medication data in networked research. J Biomed Inform. 2014:52:4-10

48. Kharrazi H, Lasser EC, Yasnoff WA, et al. A proposed national research and development agenda for population health informatics: summary recommendations from a national expert workshop. J Am Med Inform Assoc. 2017;24(1):2-12.
49. Kharrazi H, Gonzalez CP, Lowe KB, et al. Forecasting the maturation of electronic health record functions among U.S. hospitals: retrospective analysis and predictive model. J Med Internet Res. 2018;20(8):e10458.

50. Gamache R, Kharrazi H, Weiner JP. Public and population health informatics: the bridging of big data to benefit communities. Yearb Med Inform. 2018;27(1):199-206.

51. Kharrazi H, Weiner JP. IT-enabled community health interventions: challenges, opportunities, and future directions. EGEMS (Wash DC) 2014;2(3): 1117.

52. Dixon B, Pina J, Kharrazi H, Gharghabi F, Richards J. What's past is prologue: a scoping review of recent public and global health informatics literature. Online J Public Health Inform. 2015;7(2):e216

53. Dixon B, Kharrazi H, Lehman H. Public health and epidemiology informatics: recent research and events. Yearb Med Inform. 2015;10(1) 199-206.

54. Patel CH, Zimmerman KM, Fonda JR, et al. Medication complexity, medication number, and their relationships to medication discrepancies. Ann Pharmacother. 2016;50(7):534-40.

55. Wimmer BC, Bell JS, Fastbom J, et al. Medication regimen complexity and polypharmacy as factors associated with all-cause mortality in older people: a population-based cohort study. Ann Pharmacother. 2016;50(2):89-95

56. Wimmer BC, Bell JS, Fastbom J, et al. Medication regimen complexity and number of medications as factors associated with unplanned hospitalizations in older people: a population-based cohort study. J Gerontol A Biol Sci Med Sci. 2016;71(6):831-37.

57. Tesfaye WH, Peterson GM, Castelino RL, et al. Medication regimen complexity and hospital readmission in older adults with chronic kidney disease. Ann Pharmacother. 2019;53(1):28-34.

58. Eisenberg DF, Placzek H, Gu T, et al. Cost and consequences of noncompliance to oral bisphosphonate treatment. J Manag Care Spec Pharm. 2015;21(1):56-65. Available at: https://www.jmcp.org/doi/10.18553/ jmcp.2015.21.1.56.

59. Cho H, Byun JH, Song I, et al. Effect of improved medication adherence on health care costs in osteoporosis patients. Medicine (Baltimore). 2018;97(30):e11470.

60. Feldman CH, Yazdany J, Guan H, et al. Medication nonadherence is associated with increased subsequent acute care utilization among Medicaid beneficiaries with systemic lupus erythematosus. Arthritis Care Res (Hoboken). 2015;67(12):1712-21.

61. Kim JA, Lim MK, Kim K, et al. Adherence to inhaled medications and its effect on healthcare utilization and costs among high-grade chronic obstructive pulmonary disease patients. Clin Drug Investig. 2018;38(4):333-40.

62. Kamath A, Kamath P, Hadigal S, Chowta MN, Sudhakar P. Complexity of medication regimen prescribed on hospital discharge in paediatric and geriatric patients with psychiatric disorder. J Young Pharm. 2017;9(3):395.

63. Barnette DJ, Hanks C, Li W, Porter K. Patient-level medication regimen complexity in an adolescent and adult population with autism spectrum disorders. Pharmacotherapy. 2019;39(6):636-44.

64. Fortin A, Morin V, Ramsay S, Gervais P, Bolduc S. Adherence to antimuscarinics in children with overactive bladder. Paediatr Child Health. 2017:22(5):255-58.

65. El-Rachidi S, Larochelle JM, Morgan JA. Pharmacists and pediatric medication adherence: bridging the gap. Hosp Pharm. 2017;52(2):124-31. 
Assessing the Population-Level Correlation of Medication Regimen Complexity and Adherence Indices Using Electronic Health Records and Insurance Claims

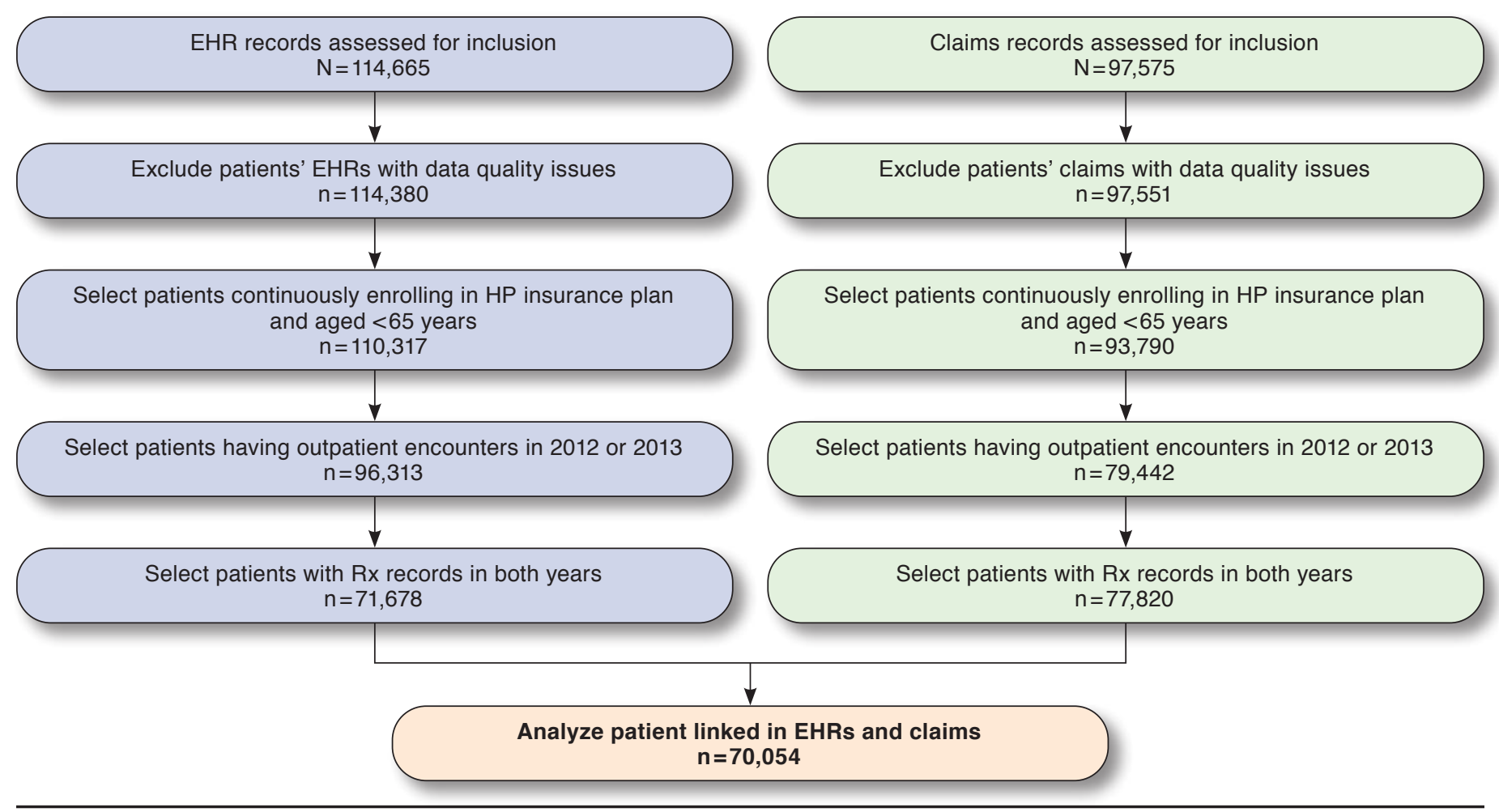

EHR=electronic health records; HP= HealthPartners [health system]; $R x=$ medication or prescription. 
Assessing the Population-Level Correlation of Medication Regimen Complexity and Adherence Indices Using Electronic Health Records and Insurance Claims

\begin{tabular}{|c|c|c|}
\hline $\begin{aligned} \text { APPENDIX B } & \text { Medi } \\
& \text { Adhe } \\
& (\mathrm{N}=\end{aligned}$ & $\begin{array}{l}\text { n Comple } \\
\text { e Indices } \\
\text { 4) }\end{array}$ & $\begin{array}{l}\text { and } \\
13\end{array}$ \\
\hline Medication Indices & EHR & Claims \\
\hline$\overline{M R C I^{\mathrm{a}}(\text { mean } \pm \mathrm{SD})}$ & $13.9 \pm 18.3$ & - \\
\hline $\mathrm{MRCI}^{\mathrm{b}}($ mean $\pm \mathrm{SD})$ & $14.1 \pm 18.7$ & - \\
\hline ICD count (mean \pm SD) & $30.3 \pm 48.4$ & $13.4 \pm 10.1$ \\
\hline$<10$ & $32,002 \quad(45.7)$ & $30,411 \quad(43.4)$ \\
\hline $10-19$ & $15,634(22.3)$ & $26,585(37.9)$ \\
\hline $20-39$ & $9,142(13.0)$ & $11,390(16.3)$ \\
\hline$>40$ & $13,230(18.9)$ & $1,665 \quad(2.4)$ \\
\hline Missing & $46 \quad(0.1)$ & $3(0.0)$ \\
\hline Prescription count ${ }^{b}($ mean \pm SD) & $4.3 \pm 3.8$ & $5.8 \pm 5.4$ \\
\hline$<3$ & $32,859(46.9)$ & $23,969(34.2)$ \\
\hline $3-10$ & $32,122(45.9)$ & $35,291(50.4)$ \\
\hline$>10$ & $4,989 \quad(7.1)$ & $10,744(15.3)$ \\
\hline $\mathrm{MPR}^{\mathrm{c}}($ mean $\pm \mathrm{SD}, \%)$ & - & $64.2 \pm 32.9$ \\
\hline$\geq 80.0$ & - & $15,547(22.2)$ \\
\hline $50.0-80.0$ & - & $17,498(25.0)$ \\
\hline$\leq 50.0$ & - & $18,950(27.1)$ \\
\hline NA & - & $18,059(25.8)$ \\
\hline $\mathrm{MPR}^{\mathrm{b}}($ mean $\pm \mathrm{SD}, \%)$ & - & $76.2 \pm 58.1$ \\
\hline$\geq 80.0$ & - & $16,987(24.2)$ \\
\hline $50.0-80.0$ & - & $14,879(21.2)$ \\
\hline$\leq 50.0$ & - & $24,101 \quad(34.4)$ \\
\hline NA & - & $14,087(20.1)$ \\
\hline 7-day PFRd $($ mean $\pm S D, \%)$ & & 3.6 \\
\hline 30-day PFRd (mean \pm SD, \%) & & 0.2 \\
\hline $\begin{array}{l}\text { Note: Values are } n(\%) \text { unless othern } \\
\text { aUsing internal ID of prescriptions. } \\
\text { bUsing NDC numbers. } \\
\text { 'Using active ingredients. } \\
\text { dUsing RxMGs. } \\
\text { ICD = International Classification of } \\
\text { MRCI = medication regimen complex } \\
\text { Drug Code; PFR = prescription fill ra } \\
\text { morbidity groups. }\end{array}$ & $\begin{array}{l}\text { ses; } M P R=\text { med } \\
\text { lex; } N A=\text { not } \\
M G=\text { prescribed }\end{array}$ & $\begin{array}{l}\text { n possession ratio; } \\
\text { le; NDC = National } \\
\text { ication-defined }\end{array}$ \\
\hline
\end{tabular}

